\title{
Zum Ovalwerk des Leonardo da Vinci.*)
}

Von Wilhelm Rulf in Wien.

Wenn die Achsen einer Ellipse gegeben sind, so kam man bekanntlich dieselbe mittelst eines Papierstreifens construieren, indem man eine Strecke von der Länge $a+b$ oder $a-b$ mit ihren Endpunkten auf den Achsen bewegt. Im ersten Falle liegt der die Ellipse beschreibende Punkt innerhalb der Strecke und zerlegt sie in $a$ and $b$, im zweiten Falle liegt er außerhalb so, dass seine Entfernungen von den Endpunkten der Strecke $a$, beziehnngsweise $b$ betragen.

Die Anführung dieser, für das technische Zeichnen wegen Vermeidung aller Hilfslinien so wichtigen Construction, hat in dem Buche A. L. B us ch: Vorschule der darstellenden Geometrie. Zweite vermehrte Auflage. Berlin, Georg Reimer 1868 auf Seite 72

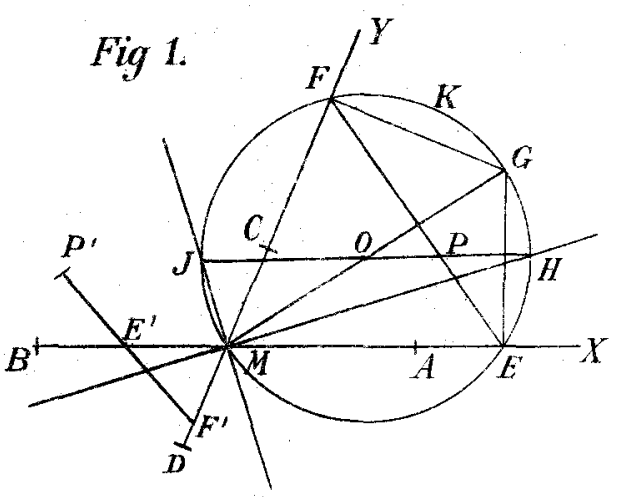
zu einem Irrthume geführt, indem im zweiten Absatze der genannten Seite gesagt wird, dass die Construction der Ellipse mittelst eines Papierstreifens auch dann anwendbar ist, wenn $A B$ and $C D$ conjugierte Diameter der Ellipse sind.

Bezeichnet man mit $M$ den Mittelpunkt der Ellipse, so erhält man zwar, wenn man mittelst des Papierstreifens erstens mit der Summe $M A+M C$ und zweitens mit der Differenz $M A-M C$ Curven construirt zwei von einander verschiedene Ellipsen, die aber schon deshalb und dann auch nicht mit der gegebenen Eilipse übereinstimmen können, da sie in $A B$ und $C D$ nicht conjugierte Diameter besitzen.

*) Siehe Holzmüller: Methodisches Lehrbuch der Elementar-Mathematik. Leipzig B. G. Teubner 1895. Dritter Theil, Seite 45. 
Zunächst soll auf elementar geometrischem Wege gezeigt werden, dass durch die Bewegnng einer constanten Strecke zwischen den Schenkeln eines beliebigen Winkels eine Ellipse erzeugt werden kann. Hiebei wird vorausgesetzt, dass diese Ellipsenentstehung für einen rechten Winkel, die sich in manchen Büchern, auch in der Form von Ellipsenzirkeln, vorfindet, bekannt ist.

Es sei Fig. 1. $x M y$ der Winkel, zwischen dessen Schenkeln sich die constante Strecke $E F$ bewegt. Hiebei sei $F P=M A$ und $P E=M C$. Dann entstehen auch bei der Bewegung der Strecke die Punkte $A, B, C$ und $D$.

Man errichte in $E$ eine Senkrechte auf $M x$, in $F$ eine solche auf $M y$. Beide treffen sich in $G$. Hierauf ist $M E G F$ ein Sehnenviereck, $K$ der demselben umschriebene Kreis. Dieser hat bei der Bewegung von $E F$ einen unveränderlichen Durchmesser, da in ihm der der Länge nach unveränderlichen Sehne $E F$ immer derselbe Umfangswinkel $x M y$ entspricht. Der rechten Winkel bei $E$ und $F$ wegen ist $M G$ ein Durchmesser des Kreises, und demnach $O$ sein Mittelpunkt, wenn $M O=O G$ ist. Verbindet man $O$ mit dem Punkte $P$, der die Curve beschreibt, so schneidet diese Verbindungslinie den Kreis $K$ in $H$ und $I$, und es lässt sich zeigen, dass die Verbindungslinien $M H$ und $M I$ bei der Bewegung ron $E F$ feste Gerade sind.

Da der Kreis $K$ bei der Bewegung von $E F$ seinen Durchmesser nicht ändert, und der Punkt $P$ dieselbe Lage in der constanten Sehne desselben beibehält, so ist $E H$ ein der Länge nach un reränderlicher Kreisbogen, daher auch sein Peripheriewinkel $x M H$ constant, demnach $M H$ eine fixe Gerade, so wie die auf ihr senkrecht-stehende $M I$.

Bei der Bewegung von $E F$ behält aber auch $O P$ beständig dieselbe Länge, daher ist $P$ ein bestimmter Punkt des Durchmessers $H I$. Es bewegt sich also jetzt eine constante Strecke $H I$ zwischen den Schenkeln des rechten Winkels $H M I$, und $P$ beschreibt daher eine Ellipse.

Genau so wird der Beweis geliefert, wenn $E^{\prime} F^{\prime}=E^{\prime} P^{\prime}-$ $-E^{\prime} P^{\prime}=M A-M C$ die constante Strecke ist.

Um nun zu zeigen, dass die durch $E F$ und $E^{\prime} F^{\prime}$ erzeugten Ellipsen nicht identisch sind, wird folgende Aufgabe gelöst:

"Die Schnittpunkte beider so erzeugten Ellipsen sind mit einer zu $M x$ oder $M y$ parallelen Geraden zu construieren “.

Es sei Fig. 2. $L$ eine zu $M y$ parallele Gerade. Der um $M$ mit $M A$ beschriebene Kreis schneidet $L$ in 1 und 2. Man ziehe den Strahl $M 1$ und mache auf demselben $13=14=M C$. Durch 3 und 4 ziehe man Parallele zu $M y$, welche $M x$ in 5 und 6 schneiden. Zieht man durch 5 Parallele zu $M 1$ und $M 2$, so schneiden diese $L$ in I and II, zwei Punkten, welche der mit $E^{\prime} F^{\prime \prime}$ erzeugten Ellipse entsprechen, denn sehneidet z. B. 5I. . My in 7, so ist, wie 
man leicht aus dem Parallelogramm ersieht, $7 \mathrm{I}=M A$ und $5 \mathrm{I}=M C$.

Die durch 6 zu $M 1$ und $2 M$ gezogenen Parallelen schneiden $L$ in den Punkten I' und II', welche, wie man auf ähnliche Weise rekennt, der mit $M F$ beschriebenen Ellipse entsprechen.

Bezeichnet man den Schnittpunkt von $L$ mit $M x$ mit $N$, so finden der Parallelen wegen die Proportionen statt:

$$
\begin{aligned}
& 5 \mathrm{I}: M 1=5 N: M N \\
& 5 \mathrm{II}: M 2=5 N: M N
\end{aligned}
$$

aus welchen $5 \mathrm{I}=5 \mathrm{II}$ folgt, da auch $M L=M 2$ ist. Wenn nun II' mit I und II mit I' zusammenfallen würde, so müsste das Viereck 5I 6II ein Rhombus sein, was nicht möglich ist, da die Diagonalen dieses Viereckes sich nicht rechtwinkelig schneiden. Also sind die zwei, mit $E F$ und $E^{\prime} F^{\prime}$ erzeugten Ellipsen nicht indentisch. Sie fallen erst dann zusammen, wenn $M y$ senkrecht steht auf $M x$.

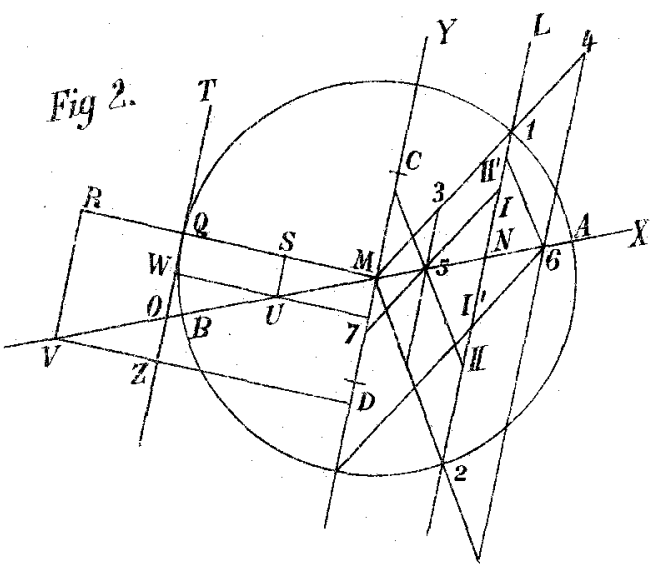

Zieht man die Parallelle $L$ durch $A$, so wird sie von dem mit $M A$ beschriebenen Kreise noch einmal außer in $A$ geschnitten. Es wird sich also auf ihr noch je ein Schnittpunkt mit einer jeden der zwei Ellipsen erge. ben. In Folge dessen können $A B$ und $C D$ nicht cojugierte Diameter sein, wodurch der Irrthum in dem Buche von Busch erwiesen erscheint.

Man ziehe ferner Fig. 2 an den mit $M A$ beschriebenen Kreis die Tangente $T$, deren Berührungspunkt $Q$ sei. Man mache $R Q=$ $=Q S=M C$ und ziehe durch $R$ und $S$ Parallele zu $M y$, welche $M x$ in $V$ und $U$ treffen. Fallt man ferner von $V$ und $U$ Senkrechte auf $T$, deren Fußpunkte $Z$ und $W$ sind, so sind $Z$ und $W$ nach Vorhergehendem die Berührungspunkte der Geraden $T$ mit den zwei Ellipsen. Daraus ergibt sich der Satz:

„Die vier gemeinschaftlichen Tangenten der beiden Ellipsen bilden ein mit diesen concentrisches Parallelogramm, dessen Seiten zu $M x$ und $M y$ parallel sind und dessen Höhen an Länge gleich $A B$ und $C D$ sind."

Ist $O$ der Schnittpunkt der verlängernten $A B$ mit $T$, so liegt $O$ in der Mitte zwischen den Ellipsenberührungspunkten, also es ist $O Z=O W$. 
Aus den ähnlichen Dreiecken $O W U$ und USM folgt die Proportion:

$$
O W: U S=Q S: S M \text {. }
$$

Aus den ähnlichen Dreiecken $V O Z$ und $U S M$ jene:

$$
O Z: U S=V Z: S M \text {. }
$$

Da nun $V Z=R Q=Q S$, so folgt aus den Proportionen

$$
O W=O Z \text {. }
$$

